\title{
Diabetes Insipidus: New Concepts for Diagnosis
}

\author{
Mirjam Christ-Crain ${ }^{a, b}$ \\ a Department of Endocrinology, Diabetology and Metabolism University Hospital Basel, Basel, Switzerland; \\ ${ }^{b}$ Department of Clinical Research, University of Basel, Basel, Switzerland
}

\section{Keywords}

Diabetes insipidus · Primary polydipsia $\cdot$ Differential

diagnosis

\begin{abstract}
Diabetes insipidus (DI), be it from central or from nephrogenic origin, has to be differentiated from primary polydipsia. This differentiation is crucial since wrong treatment can have dangerous consequences. For decades, the "gold standard" for differential diagnosis has been the standard water deprivation test. However, this test has several limitations leading to an overall limited diagnostic accuracy. In addition, the test has a long duration of $17 \mathrm{~h}$ and is cumbersome for patients. Also clinical signs and symptoms and MRI characteristics overlap between patients with $\mathrm{DI}$ and primary polydipsia. Direct measurement of arginine vasopressin (AVP) upon osmotic stimulation was first shown to overcome these limitations, but failed to enter clinical practice mainly due to technical limitations of the AVP assay. Copeptin is secreted in equimolar ratio to AVP, mirroring AVP concentrations in the circulation. We have shown that copeptin, without prior fluid deprivation, identifies patients with nephrogenic DI. For the more difficult differentiation between central DI and primary polydipsia, a copeptin level of 4.9 $\mathrm{pmol} / \mathrm{L}$ stimulated with hypertonic saline infusion differentiates between these 2 entities with a high diagnostic accuracy and is superior to the water deprivation test. However,
\end{abstract}

it is important to note that close and regular sodium monitoring every $30 \mathrm{~min}$ during the hypertonic saline test is a prerequisite, which is not possible in all hospitals. Furthermore, side effects are common. Therefore, a nonosmotic stimulation test would be advantageous. Arginine significantly stimulates copeptin and therefore is a novel, so far unknown stimulus of this peptide. Consequently, infusion of arginine with subsequent copeptin measurement was shown to be an even simpler and better tolerated test, but head to head comparison is still lacking.

(c) 2020 S. Karger AG, Basel

\section{Introduction}

Diabetes insipidus (DI) is part of the so-called polyuria polydipsia syndrome. This syndrome is defined by an output of $>50 \mathrm{~mL} / \mathrm{kg}$ body weight per $24 \mathrm{~h}$ of hypotonic urine $\left(<300 \mathrm{mOsm} / \mathrm{kg} \mathrm{H}_{2} \mathrm{O}\right)$, which is accompanied by polydipsia of $>3 \mathrm{~L}$ a day [1]. The differential diagnosis of hypotonic polyuria includes central or nephrogenic DI on 1 hand and primary polydipsia on the other hand. A correct differential diagnosis is important since treatment differs and application of the wrong treatment may lead to deleterious clinical consequences for the patients, such as water intoxication if treatment with Desmopressin is applied to patients with primary polydipsia.
Mirjam Christ-Crain, $\mathrm{MD}, \mathrm{PhD}$ 
The "gold standard" for differential diagnosis for decades has been the classical, standard water deprivation test. This test, however, has several limitations leading to a rather low diagnostic accuracy of only around $70 \%$. Therefore, Zerbe and Robertson [2] proposed in the 1980s to directly measure arginine vasopressin (AVP) upon osmotic stimulation with hypertonic saline. Initial results looked promising, but unfortunately, measurement of AVP did not enter clinical practice, mainly due to technical limitations of the AVP assay and due to the fact that reliable assays are mostly not commercially available. In this review, we will therefore discuss new concepts for the diagnosis and differential diagnosis of the polyuria polydipsia syndrome.

\section{The Polyuria Polydipsia Syndrome}

The polyuria polydipsia syndrome includes central or nephrogenic DI on 1 hand and primary polydipsia on the other hand [3]. It is not uncommon in clinical practice with increasing prevalence especially since many lifestyle programs suggest that consuming several liters of fluids a day is generally healthy.

DI leads to hypotonic polyuria which is usually accompanied by subsequent polydipsia. In central DI, there is an insufficient secretion of AVP from the posterior pituitary $[4,5]$. Central DI is most often induced by lesions of the posterior pituitary or the hypothalamic median eminence. The most common acquired causes are trauma, surgery of the pituitary, neoplastic, vascular, autoimmune, infectious, or granulomatous diseases. Pituitary surgery leads to central DI in up to $30 \%$ of cases, which is most often of transient nature. Permanent postsurgical DI is much less common and only occurs in $2-10 \%$ [6]. Central DI can also be inherited, however, inherited forms are rather rare $[7,8]$. As mentioned above, in most cases, thirst mechanisms are intact, therefore leading to a subsequent polydipsia. If thirst mechanisms are also impaired, as happens in the so-called osmoreceptor dysfunction, lack of polydipsia may lead to hyperosmolality and dehydration which can have clinically serious complications $[3,9]$.

In contrast, in nephrogenic DI, AVP levels are normally secreted, but there is a resistance toward the action of AVP at the level of the kidneys [10]. Nephrogenic DI is also most often acquired, with the best known cause being drug-induced nephrogenic DI. Mainly lithium intake is known to induce nephrogenic DI [10]. Furthermore, it can also be inherited, with mu- tations the key proteins of AVP receptor 2 and aquaporin 2.

In primary polydipsia, AVP secretion and renal action are not affected. The primary problem is an excessive fluid intake over a longer period of time. Very rarely, it can result from an abnormality in the thirst center (in which case it is called dipsogenic DI). However, much more often, it is seen in different psychiatric disorders (called psychogenic polydipsia). Subsequent to the excessive fluid intake osmolality and AVP synthesis and release are suppressed. The consequence is excretion of free water. If polydipsia is happening over an extended period of time, it can result in reno-physiological adaptions, including first a downregulation of the aquaporin 2 channels in the kidneys and second compromising the renal medullary concentration gradient. Both these processes are factors limiting urinary concentration capacity. Clear diagnostic distinction between the different forms of DI and primary polydipsia is critical as causal treatment obviously varies and application of the wrong treatment can be clinically harmful and potentially life-threatening [11].

\section{Differential Diagnosis by Water Deprivation Test}

For many years, the standard diagnostic test for the evaluation of polyuria-polydipsia syndrome was the classical water deprivation test [5]. With this test, insufficient AVP secretion or effect is diagnosed upon insufficient concentration capacity of the kidneys over osmotic stimulation which is reached with a prolonged period of thirsting (usually $16 \mathrm{~h}$ ) and its response to exogenous AVP administration (Desmopressin) [12-14]. The classical interpretation of the test is based on results on data from Miller et al. [5]. In this study, 29 patients with central DI (11 with a partial DI), 2 patients with nephrogenic DI and 5 patients with primary polydipsia were evaluated. Patients with a urinary osmolality below $300 \mathrm{mOsm} /$ $\mathrm{kg}$ during the water deprivation test are diagnosed to have complete DI. If these patients increase with their urinary osmolality $>50 \%$ after exogenous AVP administration, the final diagnosis is complete central DI, and conversely, if their urinary osmolality after exogenous AVP administration does increase $<50 \%$, the final diagnosis is complete nephrogenic DI. In those patients in whom urinary osmolalities increased to values between 300 and 800 $\mathrm{mOsm} / \mathrm{kg}$ upon water deprivation, partial central DI, or primary polydipsia is present. Partial central DI patients increased upon exogenous AVP administration $>9 \%$, whereas patients with primary polydipsia increased $<9 \%$. 
Fig. 1. AVP and its protein products. The prohormone is packaged into neurosecretory granules of magnocellular neurons. During axonal transport of the granules from the hypothalamus to the posterior pituitary, enzymatic cleavage of the prohormone generates the final products: AVP, neurophysin and the $\mathrm{COOH}$-terminal glycoprotein copeptin. Adapted from [3].
Pre-provasopressin

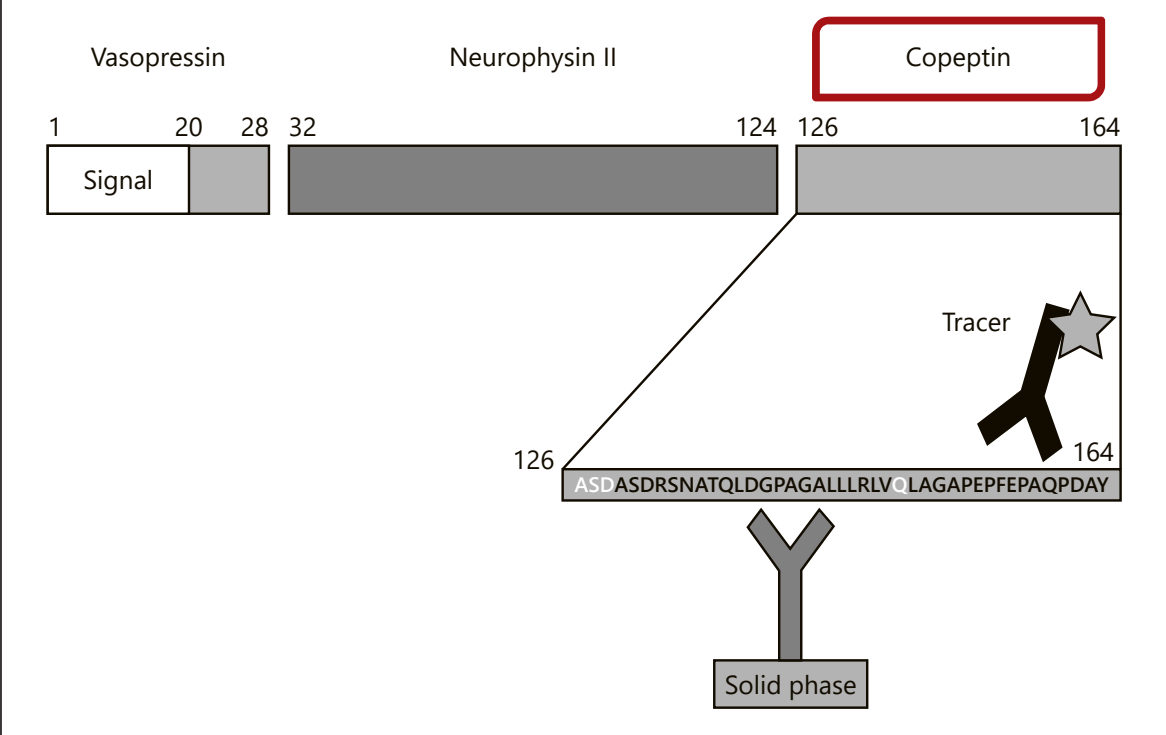

However, these cutoff values derive from only one single post hoc analysis of this small cohort of patients and as evident in the raw data show a quite wide overlap in urinary osmolality levels [5]. Recent data in fact, aiming to validate these findings, showed a diagnostic accuracy of using these criteria in the classical water deprivation test of only around $70 \%$, with an especially low diagnostic accuracy in patients with primary polydipsia $[12,14]$.

To improve the differential diagnosis of the polyuria polydipsia syndrome, Zerbe and Robertson [2] proposed the direct test, where plasma AVP is measured upon osmotic stimulation not only by thirsting, but by stimulation with hypertonic saline infusion. AVP-levels are then interpreted in relation to the area of normality describing the physiological relationship between AVP release and plasma osmolality. Patients with osmotically stimulated plasma AVP levels above the area of normality are diagnosed as nephrogenic DI, patients with levels below the area of normality as central DI, and patients with levels within the normal area as primary polydipsia $[2,7]$. Reassuringly, the results showed that direct measurement of plasma AVP has the potential to improve the diagnostic accuracy of the classical "indirect" water deprivation test with interpretation of urinary osmolality levels. However, despite these promising data, this direct test based on AVP measurement did not enter everyday clinical routine. Unfortunately, recent studies

Diabetes Insipidus: New Concepts for Diagnosis failed to confirm these promising data when using commercially available AVP assays. Specifically, with commercially available assays, a correct diagnosis was only reached in $38 \%$ of patients and a diagnostic accuracy was especially low in the differentiation between partial central DI and primary polydipsia [12]. The problem is that an accurate definition of the normal physiological relationship describing plasma AVP as a function of osmotic activity has long been missed [15], but is an important condition for the use of direct AVP measurement [12]. Furthermore, the AVP assay per se has several technical limitations, resulting in a high preanalytical instability $[1,16,17]$. Of note, the few reliable assays are not commercially available.

Therefore, new concepts for differential diagnosis are urgently needed.

\section{Copeptin: A New Surrogate Marker for AVP}

Copeptin was originally detected in 1972 in the posterior pituitary of pigs $[18,19]$, and it derives from the 164 amino acid precursor protein Pre-Pro-Vasopressin together with AVP and Neurophysin II. It is a 39 amino acid long glycosylated peptide with a leucine-rich core region $[19,20]$ and has a molecular mass of around $5 \mathrm{kDa}[21]$ (Fig. 1). 
Its physiological function until today remains largely unknown.

Copeptin strongly correlates with plasma AVP with a correlation index of $r=0.8$ [22]. Of note, the correlation of plasma copeptin with plasma osmolality was even stronger than the correlation of AVP with plasma osmolality [23], most likely due to the complexity and methodological drawbacks of the AVP assay.

Processed from the same precursor peptide, the release of plasma copeptin and plasma AVP into circulation is regulated by the same physiological stimuli, which is a relative increase in systemic osmolality and a relative decrease in arterial blood volume and pressure $[22,24]$. The surrogate properties of copeptin for physiological AVP release secondary to osmotic regulation was first shown in a study including 24 healthy adults, where fluid deprivation as well as hypertonic saline infusion led to a significant increase in plasma copeptin levels [23, 24].

There are also nonosmotic stimuli for AVP and copeptin such as nausea, hypovolemia, and hypotension as well as unspecific somatic stress as seen in, for example, ischemic stroke, myocardial infarction, or pneumonia [25-27].

In contrast to AVP, copeptin can be measured in clinical routine with commercially available assays with a high-standard technical performance. Two assays are currently available and validated, on 1 side the original manual sandwich immunoluminometric assay [21] and on the other side the automated immunofluorescent successor (on the KRYPTOR platform). Main advantages of measuring copeptin as compared to AVP are that it requires only a small sample volume $(50 \mu \mathrm{L}$ of serum or plasma), no extraction step, or other preanalytical procedures, and that results are normally available in $<2 \mathrm{~h}$. Moreover, copeptin is much more stable in plasma or serum ex vivo with $<20 \%$ loss of recovery for at least 7 days at room temperature and at 14 days at $4{ }^{\circ} \mathrm{C}$ making the handling of patient blood samples less complicated.

Based on the above, copeptin could be an attractive new surrogate marker for the diagnosis and differential diagnosis of DI.

\section{First Concept: Baseline Copeptin to Identify Nephrogenic DI and Hypertonic Saline Infusion Plus Copeptin Measurement to Diagnose DI}

Based on the fact that osmotic stimulation is the strongest and best known stimulus for AVP and copeptin release, we first studied copeptin for the differential diagnosis of the polyuria polydipsia syndrome upon osmotic stimulation. We used a combined water deprivation test followed by $3 \%$ saline infusion. With this procedure, the aim was to increase plasma sodium levels to above 147 $\mathrm{mmol} / \mathrm{L}$ which is only rarely reached by water deprivation alone, especially in patients with primary polydipsia or mild forms of DI. In 55 patients with nephrogenic or central DI or primary polydipsia [13], the first finding was that in patients with nephrogenic DI a single baseline copeptin level of $>21.4 \mathrm{pmol} / \mathrm{L}$ without prior thirsting identified all patients with nephrogenic diabetes.

As expected, however, baseline copeptin values in all other diagnoses (i.e., central DI and primary polydipsia) largely overlapped. The second finding was that upon osmotic stimulation, a copeptin level of $>4.9 \mathrm{pmol} / \mathrm{L}$ differentiated patients with central DI from patients with primary polydipsia with a high diagnostic accuracy of $96 \%$. AVP was also measured in this study, with a validated assay, showing a slightly lower diagnostic accuracy [13].

We then validated this copeptin cutoff of $4.9 \mathrm{pmol} / \mathrm{L}$ in an international multicenter study including $156 \mathrm{pa}-$ tients with the polyuria polydipsia syndrome [14]. We further aimed to simplify the test protocol by omitting the thirsting period and by using only the hypertonic saline infusion, with the aim to increase plasma sodium levels to at least $150 \mathrm{mmol} / \mathrm{L}$. Toward this aim, hypertonic saline was initially given as a bolus dose, followed by a continuous infusion rate of $0.15 \mathrm{~mL} / \mathrm{kg} / \mathrm{min}$ until the serum sodium concentration exceeded $\geq 150 \mathrm{mmol} / \mathrm{L}$. At this time point, copeptin was measured, and serum osmolality was normalized by glucose infusion and standardized fluid intake [14]. The results showed that $97 \%$ of the patients were correctly diagnosed with the predefined copeptin cutoff level of $>4.9 \mathrm{pmol} / \mathrm{L}$ (Fig. 2). The diagnostic accuracy was similar in the differential diagnosis of patients with partial DI and patients with primary polydipsia with a correct diagnosis in $95 \%$.

The high diagnostic accuracy is reassuring - however, it is important to note that hypertonic saline infusion requires close and regular monitoring of sodium levels every $30 \mathrm{~min}$ to ensure increase of plasma sodium levels into the hyperosmotic range $[4,28]$ while preventing osmotic overstimulation [14]. Also rapid normalization of sodium levels after the osmotic stimulation is crucial to guarantee the safety of the test [14].

Based on these results, it was concluded that the hypertonic saline test plus copeptin measurement might replace the classical water deprivation test in the future differential diagnosis of hypotonic polyuria [29].

Side effects such as headache, vertigo, and malaise were more common during the hypertonic saline infusion test 


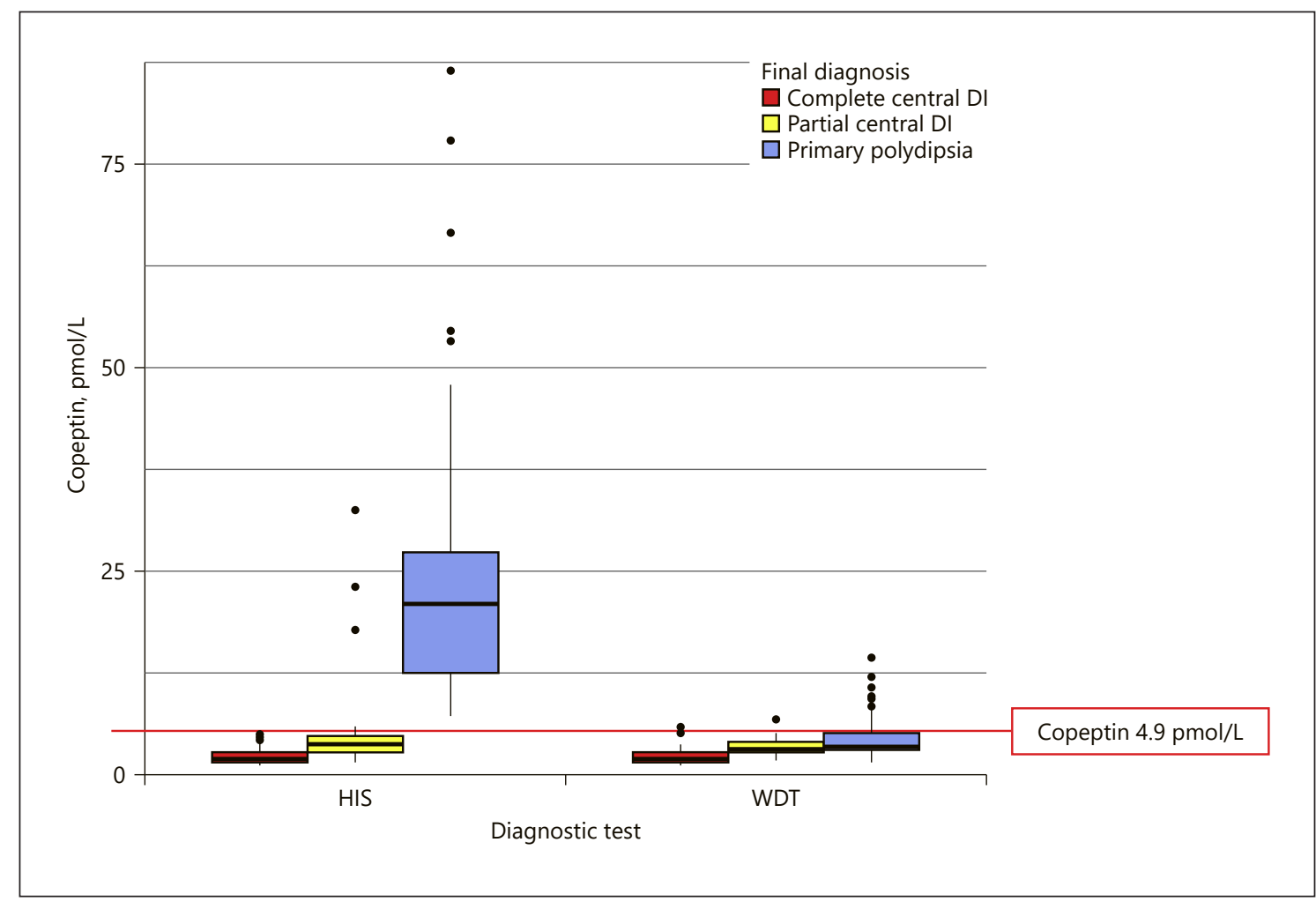

Fig. 2. Copeptin after hypertonic saline infusions compared to the classical water deprivation test in patients with complete or partial central DI or primary polydipsia [14]. DI, diabetes insipidus; HIS, hypertonic infusion of saline; WDT, water deprivation test.

than with the classical water deprivation test. Still, the majority of patients preferred the hypertonic saline stimulation with copeptin measurement over the standard water deprivation test. Most probably, the reason was the clearly shorter test duration (approximately $2 \mathrm{~h}$ for the hypertonic saline test versus $17 \mathrm{~h}$ for the water deprivation test) [14].

However, although these results are very reassuring in terms of diagnostic accuracy, the hypertonic saline infusion test is based on the induction of hypernatremia and has, therefore, several caveats, as just mentioned above: the rise in sodium can be associated with adverse effects and the test requires therefore close monitoring of sodium levels.

Therefore, a nonosmotic stimulus without the need to induce hyperosmolality would be more favorable.

\section{Second Concept: Nonosmotic Stimulation with Arginine Plus Copeptin Measurement to Diagnose DI}

Arginine is known to stimulate various hormones secreted by the anterior pituitary gland such as prolactin $[30,31]$ and growth hormone [32]. Arginine stimulation is, therefore, widely used as a simple and well-tolerated tool to diagnose growth hormone deficiency $[33,34]$, especially in children [35].

In accordance with the effects of other growth hormone secretagogues (e.g., hexarelin) [36, 37], we therefore hypothesized that arginine would also stimulate the posterior pituitary (vasopressin/copeptin) and might therefore provide a simple and alternative diagnostic test in the differential diagnosis of DI. We first showed that arginine indeed is a novel nonosmotic stimulus in healthy adults and children: in healthy adults, median (interquartile range) baseline copeptin levels were $5.2 \mathrm{pmol} / \mathrm{L}$ (3.3$10.9)$ and increased within the first $60 \mathrm{~min}$ after arginine stimulation to $9.8 \mathrm{pmol} / \mathrm{L}(6.4-19.6 ; p<0.001)$ and plateaued thereafter. In children, copeptin levels increased from baseline $4.3 \mathrm{pmol} / \mathrm{L}$ (3.2-6.0) to $6.5 \mathrm{pmol} / \mathrm{L}$ (4.7$8.5 ; p<0.001)$ within the first $60 \mathrm{~min}$ after arginine stimulation and decreased thereafter.

In patients with polyuria polydipsia syndrome, the highest diagnostic accuracy for differentiating between DI and primary polydipsia was observed for a copeptin cutoff of 3.8 $\mathrm{pmol} / \mathrm{L}$, measured at $60 \mathrm{~min}$ after arginine stimulation, $93 \%$ 
Fig. 3. Copeptin after arginine infusion, in patients with complete or partial central DI and in primary polydipsia. Adapted from [40]. DI, diabetes insipidus.

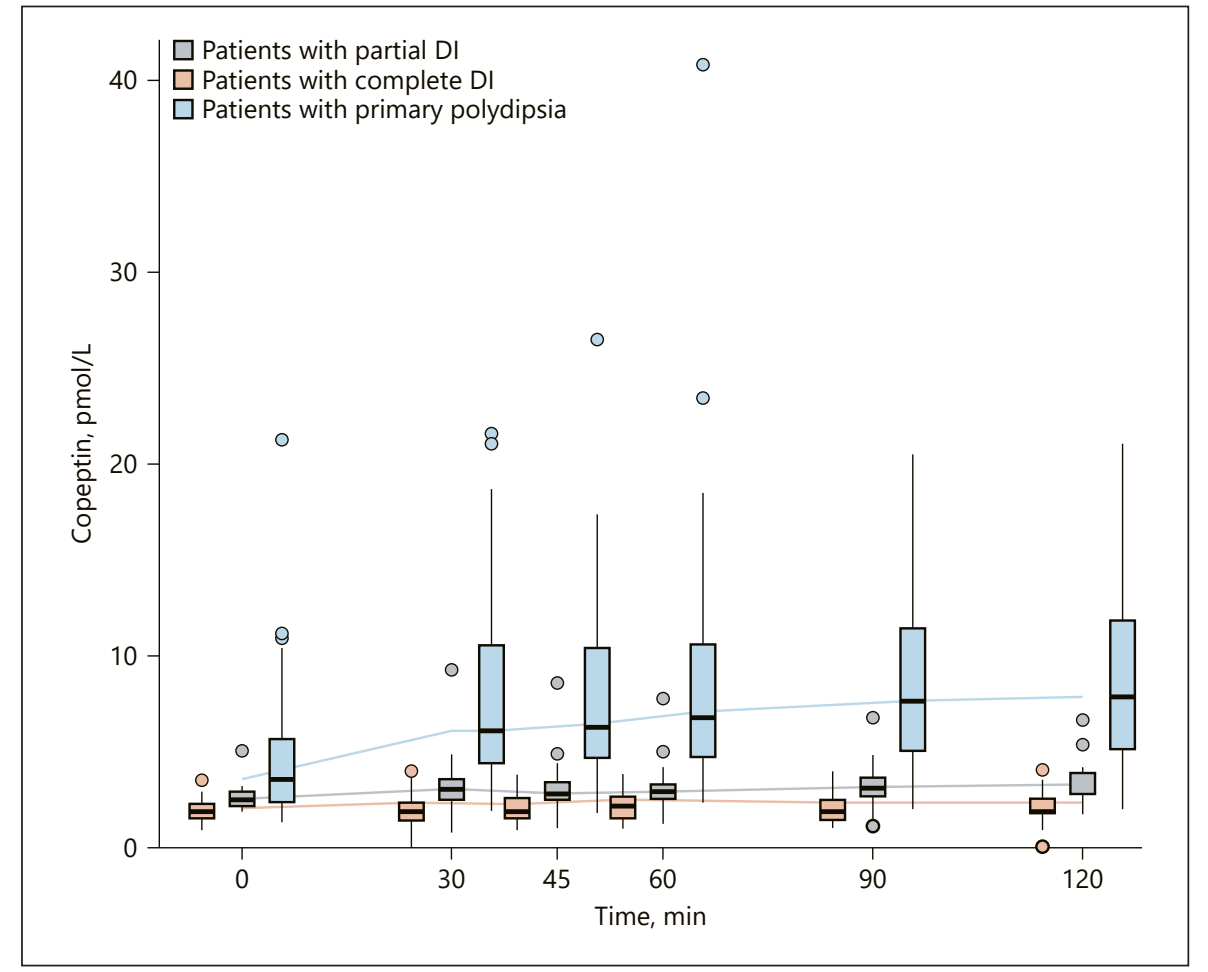

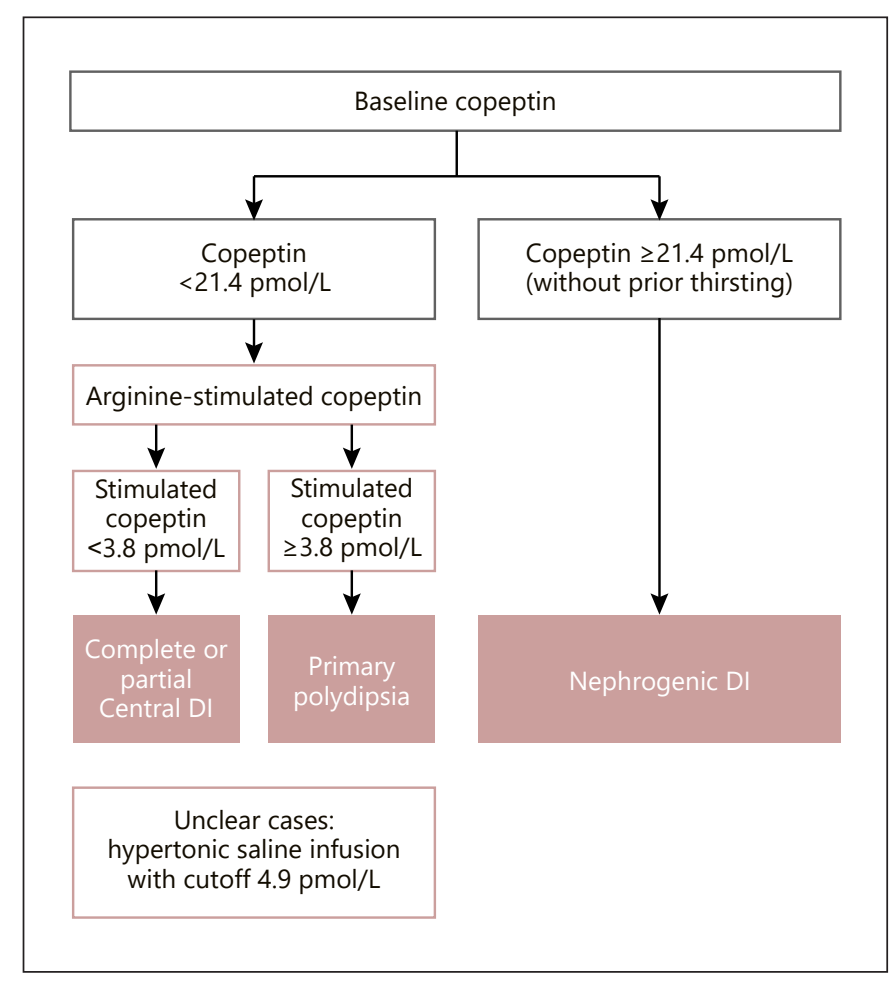

Fig. 4. Proposed two-step algorithm for the differential diagnosis of polyuria polydipsia syndrome. DI, diabetes insipidus.
(95\% CI 86-97), with a sensitivity of $93 \%$ and a specificity of $92 \%$. The area under the curve for the corresponding receiver operating characteristic was 0.95 (95\% CI 0.92-0.99). Likewise, the highest diagnostic accuracy for differentiating between partial DI and primary polydipsia was found for a copeptin cutoff of $3.8 \mathrm{pmol} / \mathrm{L}$, measured at $60 \mathrm{~min}$ after arginine stimulation, 90\% (95\% CI 82-96), with a sensitivity of $93 \%$ and a specificity of $80 \%$. The area under the curve for the corresponding receiver operating characteristic was 0.91 (95\% CI 0.83-0.99; Fig. 3).

During arginine stimulation, clinical and laboratory parameters remained generally stable and within the normal range. Overall, the test burden was rated to be low, although nausea was a frequent symptom during arginine stimulation. Compared to hypertonic saline stimulation, which is associated with adverse effects in the majority of patients [14], the tolerability profile of arginine stimulation is clearly more attractive.

However, to be able to draw inference about the comparative performance of both tests, a prospective head-tohead evaluation is currently being performed.

At the moment, a step-wise approach may be recommended, with the easier arginine stimulation test as first test and the hypertonic saline infusion test in unclear cases as the second test. A respective algorithm is proposed in Figure 4. 
Fig. 5. Copeptin in the diagnosis of postsurgical DI. DI, diabetes insipidus.

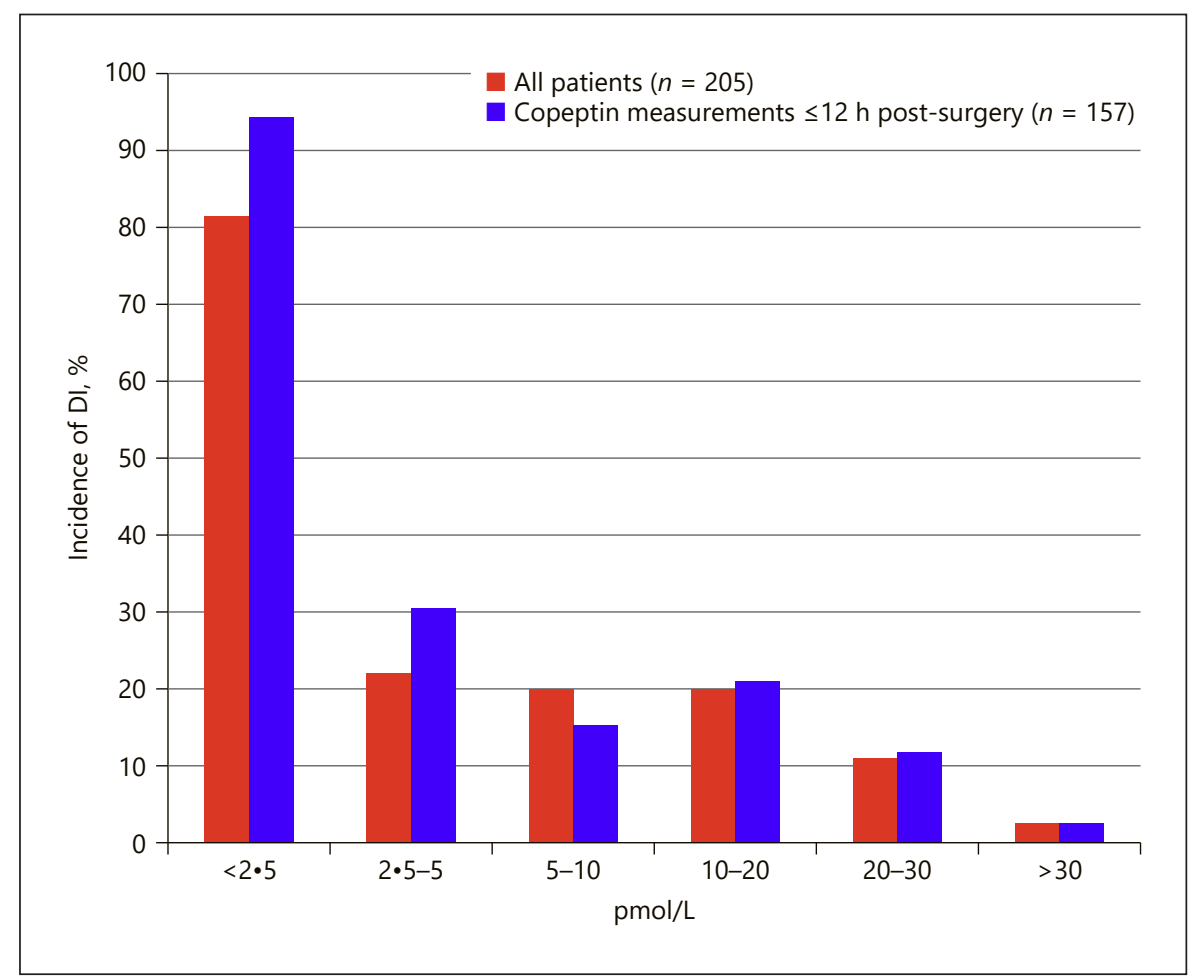

Third Concept: Nonosmotic Stress-Induced Copeptin to Diagnose Postsurgical DI

In a proof-of-concept study in 2007, it was shown that in patients after pituitary surgery, an insulin tolerance test inducing hypoglycemia and therefore a nonosmotic stress led to a significant induction of copeptin levels in those patients with an intact posterior pituitary function, but copeptin levels remained low in patients with postsurgical DI [38]. Copeptin levels of patients with intact posterior pituitary showed a maximal increase to $11.1 \pm 4.6$ $\mathrm{pmol} / \mathrm{L}$, while copeptin levels in patients with central DI remained low upon hypoglycemia at $3.7 \pm 0.7 \mathrm{pmol} / \mathrm{L}$. A hypoglycemic stimulated copeptin level $<4.75 \mathrm{pmol} / \mathrm{L}$ had an optimal diagnostic accuracy to detect central DI of $100 \%$.

Clearly, however, induction of hypoglycemia is not an attractive test for differential diagnosis of polyuria polydipsia syndrome and is not feasible as a routine test since it can be associated with severe hypoglycemia and is contraindicated in patients with cardiovascular disease or seizure history and despite its reliable diagnostic performance, the insulin tolerance test is also not appropriate in the immediate postoperative recovery phase.

However, surgery - itself known as a stressful event stimulating hypothalamic stress hormone release in-

Diabetes Insipidus: New Concepts for Diagnosis cluding AVP [39], - can be used as a "stress test" to assess functionality of AVP and copeptin secretion. In a prospective multicenter trial including 205 patients undergoing pituitary surgery, $24 \%$ of patients developed postoperative central DI. Those patients had significantly lower copeptin levels on the first postoperative day compared to patients without postoperative DI. The post hoc derived copeptin cutoff level of $<2.5$ $\mathrm{pmol} / \mathrm{L}$ had a positive predictive value for development of central DI of $81 \%$ and a specificity of $97 \%$, while a level $>30 \mathrm{pmol} / \mathrm{L}$ excluded it with a negative predictive value of $95 \%$ and a sensitivity of $94 \%$ (Fig. 5). Accordingly, copeptin measurement after pituitary surgery is helpful to predict the onset of central DI, allowing earlier targeted therapeutic measures. Even though measurement of urine volume and osmolality can identify patients with postoperative DI in most cases, these findings have implications especially in view of the nowadays often early discharge of patients after pituitary surgery. Based on our findings, copeptin measurement in patients with pituitary surgery may identify patients benefitting from closer inpatient observation and patients in whom early hospital discharge is safely possible. Of note, copeptin is only helpful for risk stratification if the value is either very low $(<2.5 \mathrm{pmol} / \mathrm{L})$ or very high (>30 pmol/L). 


\section{Conclusions}

In conclusion, the diagnosis and differential diagnosis of DI has long been made by the cumbersome water deprivation test, which has a limited diagnostic accuracy. The direct test using hypertonic saline infusion plus AVP measurement did not enter clinical routine mainly due to technical limitations of the AVP assay. Copeptin is a stable surrogate marker of AVP and provides a valuable and reliable diagnostic marker in the differential diagnosis of the polyuria-polydipsia syndrome. In patients with hypotonic polyuria, measurement of basal copeptin levels identifies nephrogenic DI. In all other patients, copeptin measurement after osmotic stimulation with $3 \%$ saline solution with the aim to increase plasma sodium level $\geq 150 \mathrm{mmol} / \mathrm{L}$ is recommended. Importantly, close monitoring of plasma sodium levels [14]. An even simpler and better tolerated alternative without the need to induce hypernatremia is the arginine stimulation test. In patients after pituitary surgery, a stress-induced high copeptin level immediately after surgery merely excludes later DI, whereas a low levels is highly predicting for later DI.

\section{Disclosure Statement}

M.C.-C.: received speaker honoraria from Thermofisher AG, the manufacturer of the Copeptin assay.

\section{Funding Sources}

M.C.-C. was supported by a grant from the Swiss National Science Foundation (SNF-162608) and the University Hospital Basel, Switzerland.

\section{References}

1 Robertson GL. Diabetes insipidus. Endocrinol Metab Clin North Am. 1995 Sep;24(3): 549-72.

2 Zerbe RL, Robertson GL. A comparison of plasma vasopressin measurements with a standard indirect test in the differential diagnosis of polyuria. N Engl J Med. 1981 Dec; 305(26):1539-46.

3 Christ-Crain M, Fenske W. Copeptin in the diagnosis of vasopressin-dependent disorders of fluid homeostasis. Nat Rev Endocrinol. 2016 Mar;12(3):168-76.

4 Robertson GL. The regulation of vasopressin function in health and disease. Recent Prog Horm Res. 1976;33:333-85.

5 Miller M, Dalakos T, Moses AM, Fellerman $\mathrm{H}$, Streeten DH. Recognition of partial defects in antidiuretic hormone secretion. Ann Intern Med. 1970 Nov;73(5):721-9.

6 Nemergut EC, Zuo Z, Jane JA Jr, Laws ER Jr. Predictors of diabetes insipidus after transsphenoidal surgery: a review of 881 patients. J Neurosurg. 2005 Sep;103(3):448-54.

7 Babey M, Kopp P, Robertson GL. Familial forms of diabetes insipidus: clinical and molecular characteristics. Nat Rev Endocrinol. 2011 Jul;7(12):701-14

8 Birk J, Friberg MA, Prescianotto-Baschong C, Spiess M, Rutishauser J. Dominant pro-vasopressin mutants that cause diabetes insipidus form disulfide-linked fibrillar aggregates in the endoplasmic reticulum. J Cell Sci. 2009 Nov;122(Pt 21):3994-4002.

9 Thompson CJ, Baylis PH. Thirst in diabetes insipidus: clinical relevance of quantitative assessment. Q J Med. 1987 Oct;65(246):853-62.

10 Bockenhauer D, Bichet DG. Pathophysiology, diagnosis and management of nephrogenic diabetes insipidus. Nat Rev Nephrol. 2015 Oct;11(10):576-88.
11 Fenske W, Allolio B. Clinical review: Current state and future perspectives in the diagnosis of diabetes insipidus: a clinical review. J Clin Endocrinol Metab. 2012 Oct;97(10):3426-37.

12 Fenske W, Quinkler M, Lorenz D, Zopf K, Haagen U, Papassotiriou J, et al. Copeptin in the differential diagnosis of the polydipsia-polyuria syndrome-revisiting the direct and indirect water deprivation tests. J Clin Endocrinol Metab. 2011 May;96(5):150615.

13 Timper K, Fenske W, Kühn F, Frech N, Arici B, Rutishauser J, et al. Diagnostic Accuracy of Copeptin in the Differential Diagnosis of the Polyuria-polydipsia Syndrome: A Prospective Multicenter Study. J Clin Endocrinol Metab. 2015 Jun;100(6):2268-74.

14 Fenske W, Refardt J, Chifu I, Schnyder I, Winzeler B, Drummond J, et al. A CopeptinBased Approach in the Diagnosis of Diabetes Insipidus. N Engl J Med. 2018 Aug;379(5): 428-39.

15 Baylis PH, Gaskill MB, Robertson GL. Vasopressin secretion in primary polydipsia and cranial diabetes insipidus. Q J Med. 1981; 50(199):345-58.

16 Milles JJ, Spruce B, Baylis PH. A comparison of diagnostic methods to differentiate diabetes insipidus from primary polyuria: a review of 21 patients. Acta Endocrinol (Copenh). 1983 Dec;104(4):410-6.

17 Kluge M, Riedl S, Erhart-Hofmann B, Hartmann J, Waldhauser F. Improved extraction procedure and RIA for determination of arginine8-vasopressin in plasma: role of premeasurement sample treatment and reference values in children. Clin Chem. 1999 Jan;45(1): 98-103.

18 Holwerda DA. A glycopeptide from the posterior lobe of pig pituitaries. I. Isolation and characterization. Eur J Biochem. 1972 Jul; 28(3):334-9.

19 Levy B, Chauvet MT, Chauvet J, Acher R. Ontogeny of bovine neurohypophysial hormone precursors. II. Foetal copeptin, the third domain of the vasopressin precursor. Int J Pept Protein Res. 1986 Mar;27(3):320-4.

20 Land H, Schütz G, Schmale H, Richter D. Nucleotide sequence of cloned cDNA encoding bovine arginine vasopressin-neurophysin II precursor. Nature. 1982 Jan;295(5847):299303.

21 Morgenthaler NG, Struck J, Alonso C, Bergmann A. Assay for the measurement of copeptin, a stable peptide derived from the precursor of vasopressin. Clin Chem. 2006 Jan; 52(1):112-9.

22 Balanescu S, Kopp P, Gaskill MB, Morgenthaler NG, Schindler C, Rutishauser J. Correlation of plasma copeptin and vasopressin concentrations in hypo-, iso-, and hyperosmolar States. J Clin Endocrinol Metab. 2011 Apr;96(4):1046-52.

23 Fenske WK, Schnyder I, Koch G, Walti C, Pfister M, Kopp P, et al. Release and Decay Kinetics of Copeptin vs AVP in Response to Osmotic Alterations in Healthy Volunteers. J Clin Endocrinol Metab. 2018 Feb;103(2): 505-13.

24 Szinnai G, Morgenthaler NG, Berneis K, Struck J, Müller B, Keller U, et al. Changes in plasma copeptin, the c-terminal portion of arginine vasopressin during water deprivation and excess in healthy subjects. J Clin Endocrinol Metab. 2007 Oct;92(10):3973-8.

25 Katan M, Fluri F, Morgenthaler NG, Schuetz P, Zweifel C, Bingisser R, et al. Copeptin: a novel, independent prognostic marker in patients with ischemic stroke. Ann Neurol. 2009 Dec;66(6):799-808. 
26 Reichlin T, Hochholzer W, Stelzig C, Laule K, Freidank H, Morgenthaler NG, et al. Incremental value of copeptin for rapid rule out of acute myocardial infarction. J Am Coll Cardiol. 2009 Jun;54(1):60-8.

27 Katan M, Christ-Crain M. The stress hormone copeptin: a new prognostic biomarker in acute illness. Swiss Med Wkly. 2010 Sep; 140:w13101.

28 Robertson GL, Shelton RL, Athar S. The osmoregulation of vasopressin. Kidney Int. 1976 Jul;10(1):25-37.

29 Rosen CJ, Ingelfinger JR. A Reliable Diagnostic Test for Hypotonic Polyuria. N Engl J Med. 2018 Aug;379(5):483-4.

30 Nair NP, Lal S, Thavundayil JX, Isaac I, Eugenio $\mathrm{H}$, Achim A, et al. Effect of normal aging on the prolactin response to graded doses of sulpiride and to arginine. Prog Neuropsychopharmacol Biol Psychiatry. 1985;9(5-6): 633-7.

31 Merimee TJ, Rabinowtitz D, Fineberg SE. Arginine-initiated release of human growth hormone. Factors modifying the response in normal man. N Engl J Med. 1969 Jun;280(26): $1434-8$.
32 Alba-Roth J, Müller OA, Schopohl J, von Werder K. Arginine stimulates growth hormone secretion by suppressing endogenous somatostatin secretion. J Clin Endocrinol Metab. 1988 Dec;67(6):1186-9.

33 Ghigo E, Bellone J, Aimaretti G, Bellone S, Loche S, Cappa M, et al. Reliability of provocative tests to assess growth hormone secretory status. Study in 472 normally growing children. J Clin Endocrinol Metab. 1996 Sep;81(9):3323-7.

34 Maghnie M, Cavigioli F, Tinelli C, Autelli M, Aricò M, Aimaretti G, et al. GHRH plus arginine in the diagnosis of acquired GH deficiency of childhood-onset. J Clin Endocrinol Metab. 2002 Jun;87(6):2740-4.

35 Growth Hormone Research Society; GH Research Society. Consensus guidelines for the diagnosis and treatment of growth hormone (GH) deficiency in childhood and adolescence: summary statement of the GH Research Society. J Clin Endocrinol Metab. 2000 Nov;85(11):3990-3.

36 Korbonits M, Little JA, Forsling ML, Tringali G, Costa A, Navarra P, et al. The effect of growth hormone secretagogues and neuropeptide $\mathrm{Y}$ on hypothalamic hormone release from acute rat hypothalamic explants. J Neuroendocrinol. 1999 Jul;11(7):521-8.
37 Korbonits M, Kaltsas G, Perry LA, Putignano $\mathrm{P}$, Grossman AB, Besser GM, et al. The growth hormone secretagogue hexarelin stimulates the hypothalamo-pituitary-adrenal axis via arginine vasopressin. J Clin Endocrinol Metab. 1999 Jul;84(7):2489-95.

38 Katan M, Morgenthaler NG, Dixit KC, Rutishauser J, Brabant GE, Müller B, et al. Anterior and posterior pituitary function testing with simultaneous insulin tolerance test and a novel copeptin assay. J Clin Endocrinol Metab. 2007 Jul;92(7):2640-3.

39 Widmer IE, Puder JJ, König C, Pargger H, Zerkowski HR, Girard J, et al. Cortisol response in relation to the severity of stress and illness. J Clin Endocrinol Metab. 2005 Aug; 90(8):4579-86.

40 Winzeler B, Cesana-Nigro N, Refardt J, Vogt DR, Imber C, Morin B, et al. Arginine-stimulated copeptin measurements in the differential diagnosis of diabetes insipidus: a prospective diagnostic study. Lancet. 2019 Aug; 394(10198):587-95. 